\title{
WEAK WAVE OPERATORS FOR THE NONLINEAR WAVE EQUATION
}

\author{
BY \\ A. ROBERT BRODSKY(1)
}

The purpose of this paper is a study of wave operators for the equation

$$
\square u=q u^{p}, \quad \square=\sum_{i=1}^{3} \frac{\partial^{2}}{\partial x_{i}^{2}}-\frac{\partial^{2}}{\partial t^{2}}, \quad q \geqq 0 .
$$

In [2] and [3], Strauss studied this problem when $q$ has sufficiently rapid decay at infinity. In this paper we will derive some information for the case $q \equiv 1$. The basic parts used will be the energy estimates of Strauss derived in [2] and an elementary lemma about Hilbert spaces. While the results in this paper refer to the existence of wave operators in the weak topology, they open new methods of attacking the problem in the strong topology.

1. The abstract problem. Let $H$ be a real separable Hilbert space with norm \|\| , and inner product $\langle$,$\rangle . Let U_{0}(t)$ denote a strongly continuous oneparameter group on $H$. Let $K$ be a locally Lipschitzian transformation on $H$ (possibly nonlinear) such that the integral equation

$$
\psi(t)=U_{0}(t) \psi_{0}+\int_{0}^{t} U_{0}(t-s) K(\psi(s)) d s
$$

has unique global solutions for any $\psi_{0} \in H$. Let $U_{1}(t)$ denote the map from $\psi_{0}$ to $\psi(t)$. $U_{1}(t)$ satisfies

(a) $U_{1}(0)$ is the identity,

(b) $U_{1}(t+r)=U_{1}(t) U_{1}(r)$,

(c) $U_{1}(-t)=U_{1}(t)^{-1}$.

Only (b) is nontrivial. However, this follows from the locally Lipschitzian character of $K$ and the fact that the norms of the $U_{1}(t) \psi_{0}$ are bounded subsets of $R$. The basic object of study in this paper are the limits as $|t| \rightarrow \infty$ of $U_{0}(-t) U_{1}(t) \psi$. We will show that these limits exist in the weak topology on $H$ for $\psi$ in a certain dense subset of $H$.

We will need the following assumptions.

(A1) There exists a dense set $\mathscr{D} \subset H$ and a function | |from $H$ to $[0, \infty]$ which is finite on $\mathscr{D}$ and a $p>2$ such that the following are true.

Received by the editors October 10, 1967 and, in revised form, January 31, 1968.

(1) This paper is being published posthumously. Professor Brodsky died July 10, 1968. The galley proof was proofread and corrected by members of the staff of the University of California at Los Angeles. 
(A2) If $\psi \in \mathscr{D}$,

$$
\left\|U_{1}(t) \psi\right\|^{2}+\left|U_{1}(t) \psi\right|^{p}=\|\psi\|^{2}+|\psi|^{p}
$$

for all $t$.

(A3) If $\psi \in \mathscr{D}$,

$$
\left|U_{1}(t) \psi\right|=O\left((1 /|t|)^{2 / p}\right) \text { as }|t| \rightarrow \infty .
$$

(A4) There exists a linear map $R$ of $\mathscr{D}$ into $\mathscr{D}$ such that if $\phi \in \mathscr{D}$ and $|\psi|<\infty$,

$$
|\langle\phi, K(\psi)\rangle| \leqq|R \phi||\psi|^{p-1} .
$$

(A5) If $\phi \in \mathscr{D}, U_{0}(t) \phi \in \mathscr{D}$ for all $t$ and

$$
\left|R U_{0}(t) \phi\right|=O\left((1 /|t|)^{(p-2) / p}\right) \quad \text { as }|t| \rightarrow \infty .
$$

The following theorem is basic to our argument.

THEOREM 1. Let $\left\{\psi_{t}\right\}$ be a set of elements of $H$ indexed by the real numbers (or any sequentially cofinal net). Assume there is a constant $B \geqq 0$ such that $\left\|\psi_{t}\right\| \leqq B$ for all $t$ and $\left\langle\phi, \psi_{t}\right\rangle$ converges as $t \rightarrow+\infty$ for $\phi \in \mathscr{D}$, a dense set in $H$. Then there exists a $\psi \in H$ such that $\psi_{l} \rightarrow \psi$ weakly as $t \rightarrow \infty$.

Proof. This theorem is a natural generalization of a standard theorem about Hilbert spaces. Consider the sequence $\left\{\psi_{n}\right\}, n$ integer. A simple estimate shows that $\left\{\psi_{n}\right\}$ is weakly convergent. Thus since $H$ is weakly sequentially complete there is a $\psi \in H$ such that $\psi_{n} \rightarrow \psi$ weakly. It then follows that $\psi_{t} \rightarrow \psi$ weakly. (Similarly there is a theorem for $t \rightarrow-\infty$.)

THËOREM 2. Under assumptions (A1), .., (A5), for any $\psi \in \mathscr{D}$, there are elements $\tilde{W}_{+} \psi$ and $\tilde{W}_{-} \psi$ in $H$ such that $U_{0}(-t) U_{1}(t) \psi$ converges weakly to these elements as $t$ approaches $+\infty$ and $-\infty$ respectively.

Proof. We will consider only the case $t \rightarrow+\infty$. The other case is similar. Let us first note that

$$
\left\|U_{0}(-t) U_{1}(t) \psi\right\|^{2} \leqq\|\psi\|^{2}+|\psi|^{p} .
$$

Thus by Theorem 1, it suffices to show that $\left\langle\phi, U_{0}(-t) U_{1}(t) \psi\right\rangle$ converges for all $\phi \in \mathscr{D}$. But

$$
\begin{aligned}
\mid\left\langle\phi, U_{0}(-t) U_{1}(t) \psi\right\rangle & -\left\langle\phi, U_{0}(-r) U_{1}(r) \psi\right\rangle \mid \\
& \leqq \int_{r}^{t}\left|\left\langle U_{0}(s) \phi, K\left(u_{1}(s) \psi\right)\right\rangle\right| d s, \quad t \geqq r
\end{aligned}
$$

by (1). But

$$
\left.\left|\left\langle U_{0}(s) \phi, K\left(U_{1}(s) \psi\right\rangle|\leqq| R U_{0}(s) \phi\right\}\right| U_{1}(s) \psi\right|^{p-1}
$$

by (A4) and (A2). But from (A3) and (A5) it follows that the right-hand side of (3) is

$$
O\left((1 / s)^{(3 p-4) / p}\right)
$$


Since $p$ is greater than $2,\left|\left\langle U_{0}(s) \phi, K\left(U_{1}(s) \psi\right)\right\rangle\right|$ is integrable and thus

$$
\left\langle\phi, U_{0}(-t) U_{1}(t) \psi\right\rangle
$$

converges.

Definition. $\tilde{W}_{+}$and $\tilde{W}_{-}$are called the weak forward and backward wave operators respectively. Theorem 2 states that they are defined on $\mathscr{D}$.

REMARK. Let us note the following elementary properties of $\tilde{W}_{+}$and $\tilde{W}_{-}$. For $\psi \in \mathscr{D}$,

$$
\left\|\tilde{W}_{ \pm} \psi\right\|^{2} \leqq\|\psi\|^{2}+|\psi|^{p},
$$

$$
\left|\left\langle\phi, W_{ \pm} \psi-U_{0}(-t) U_{1}(t) \psi\right\rangle\right|=O\left((1 /|t|)^{(2 p-4) / p}\right) \quad \text { for } \phi \in \mathscr{D} \text {. }
$$

CoRollary 1. $U_{0}(-t) U_{1}(t) \psi \rightarrow \tilde{W}_{ \pm} \psi$ strongly if and only if $\left\|\tilde{W}_{ \pm} \psi\right\|^{2}=\|\psi\|^{2}$ $+|\psi|^{p}$.

Proof.

$$
\begin{aligned}
\lim _{t \rightarrow \pm \infty}\left\|U_{0}(-t) U_{1}(t) \psi\right\|^{2} & =\|\psi\|^{2}+|\psi|^{p}-\lim _{t \rightarrow \pm \infty}\left|U_{1}(t) \psi\right|^{p} \\
& =\|\psi\|^{2}+|\psi|^{p} \quad \text { by (A2) and (A3). }
\end{aligned}
$$

Corollary 2. If $\psi \in \mathscr{D}$ and $U_{1}(t) \psi \in \mathscr{D}$, then $\tilde{W}_{ \pm} U_{1}(t) \psi=U_{0}(t) \tilde{W}_{ \pm} \psi$.

Proof. Let $\phi \in H$, then

$$
\begin{aligned}
\left\langle\phi, U_{0}(t) \tilde{W}_{ \pm} \psi\right\rangle & =\left\langle U_{0}(-t) \phi, \tilde{W}_{ \pm} \psi\right\rangle \\
& =\lim _{s \rightarrow \pm \infty}\left\langle U_{0}(-t) \phi, U_{0}(-s) U_{1}(s) \psi\right\rangle \\
& =\lim _{s \rightarrow \pm \infty}\left\langle U_{0}(-t) \phi, U_{0}(-s) U_{1}(s-t) U_{1}(t) \psi\right\rangle \\
& =\lim _{r \rightarrow \pm \infty}\left\langle\phi, U_{0}(-r) U_{1}(r) U_{1}(t) \psi\right\rangle \\
& =\left\langle\phi, \tilde{W}_{ \pm} U_{1}(t) \psi\right\rangle .
\end{aligned}
$$

Since $\phi$ is arbitrary we have the result.

Definition. Let $\tilde{\mathscr{D}}=\bigcup_{t \in R} U_{1}(t) \mathscr{D}$. In practice $\tilde{\mathscr{D}}=\mathscr{D}$.

If $\psi \in \tilde{\mathscr{D}}$, there exist $t \in R$ and $\psi_{0} \in \mathscr{D}$ such that $\psi=U_{1}(t) \psi_{0}$. For such $\psi$, we may define $\tilde{W}_{ \pm} \psi$ by

$$
\tilde{W}_{ \pm} \psi=U_{0}(t) \tilde{W}_{ \pm} \psi_{0}
$$

To guarantee that this makes sense, we need the following theorem.

THEOREM 3. If $\psi_{1}$ and $\psi_{2}$ are in $\mathscr{D}$ and there exists $s, t \in R$ such that $U_{1}(t) \psi_{1}$ $=U_{1}(s) \psi_{2}$ then

$$
U_{0}(t) \tilde{W}_{ \pm} \psi_{1}=U_{0}(s) \tilde{W}_{ \pm} \psi_{2} \text {. }
$$

Proof. By hypothesis, $\psi_{1}$ and $\psi_{2}=U_{1}(t-s) \psi_{1}$ are in $\mathscr{D}$. Thus by Corollary 2,

$$
\tilde{W}_{ \pm} U_{1}(t-s) \psi_{1}=\tilde{W}_{ \pm} \psi_{2}=U_{0}(t-s) \tilde{W}_{ \pm} \psi_{1}
$$

Therefore

$$
U_{0}(s) \tilde{W}_{ \pm} \psi_{2}=U_{0}(t) \tilde{W}_{ \pm} \psi_{1}
$$


The reason for looking at the wave operators is that we would like $U_{1}(t) \psi$ to behave like $U_{0}(t) W_{+} \psi$ for large $t$ and like $U_{0}(t) W_{-} \psi$ for small $t$. If the convergence is strong,

$$
\left\|U_{1}(t) \psi-U_{0}(t) W_{+} \psi\right\|=\left\|U_{0}(-t) U_{1}(t) \psi-W_{+} \psi\right\| \rightarrow 0
$$

as $t \rightarrow+\infty$. However, in the weak topology this may not happen. In fact, for the case of the nonlinear wave equation for $\psi \in \mathscr{D}, U_{1}(t) \psi$ tends weakly to zero as does $U_{0}(t) \phi$ for any $\phi \in H$. To make this precise let us add the assumption

(A6) If $\phi, \psi \in \mathscr{D},\left\langle\phi, U_{i}(t) \psi\right\rangle \rightarrow 0$ as $|t| \rightarrow \infty$ for $i=0,1$. For the case of the wave equation, the next result for $U_{0}$ follows immediately from the representation of $U_{0}(t)$ as the translation group on $L_{2}(-\infty,+\infty ; N), N$ a Hilbert space. (See [1].)

TheOREM 4. Assuming (A6), $U_{0}(t) \psi \rightarrow 0$ weakly as $|t| \rightarrow 0$ for all $\psi \in H$.

Proof. By Theorem 1, $U_{0}(t) \psi \rightarrow 0$ weakly as $|t| \rightarrow \infty$ for $\psi \in \mathscr{D}$. Thus, for all $\phi \in H, \psi \in \mathscr{D},\left\langle\phi, U_{0}(t) \psi\right\rangle \rightarrow 0$ as $|t| \rightarrow \infty$. Thus $\left\langle U_{0}(t) \phi, \psi\right\rangle \rightarrow 0$ as $|t| \rightarrow \infty$. Again by Theorem $1, U_{0}(t) \phi \rightarrow 0$ weakly as $|t| \rightarrow \infty$ for $\phi \in H$.

THEOREM 5. For $\psi \in \tilde{\mathscr{D}}, U_{1}(t) \psi \rightarrow 0$ weakly as $|t| \rightarrow \infty$.

Proof. Let $\psi_{0}$ be in $\mathscr{D}$ and $s \in R$ such that $U_{0}(s) \psi_{0}=\psi$. Thus for $\phi \in \mathscr{D}$,

$$
\left\langle\phi, U_{1}(t) \psi\right\rangle \rightarrow 0, \psi \in \tilde{\mathscr{D}}
$$

Now apply Theorem 1.

Let us conclude this section with another condition guaranteeing that $U_{0}(-t) U_{1}(t) \psi$ converges in the strong topology. This result has the advantage that the conditions imposed are on $U_{0}(t)$ rather than $U_{1}(t)$. Unfortunately, the condition is unknown for the wave equation. We will discuss this more fully in $\S 2$.

Lemma. Let $\left\{\psi_{t}\right\}, t \in[1, \infty)$ be a net in $H$ which converges weakly to zero as $t \rightarrow \infty$ and is bounded. Then $\psi_{t}$ converges strongly to zero if there exists an $\varepsilon>0$ such that $\psi \in H$,

$$
\left|\left\langle\psi, \psi_{t}\right\rangle\right|=O\left(t^{-\varepsilon}\right)
$$

as $t \rightarrow \infty$. In fact, $\left\|\psi_{t}\right\|=O\left(t^{-\varepsilon}\right)$.

Proof. Let $\gamma_{t}=t^{\varepsilon} \psi_{t}$. By assumption, the set $\left\{\gamma_{t}\right\}$ is weakly bounded. Thus by uniform boundedness, there is a constant $B$ such that $\left\|\gamma_{t}\right\| \leqq B$ for all $t \in[1, \infty)$. Thus $\left\|\psi_{t}\right\|=O\left(t^{-\varepsilon}\right)$.

Definition. Let ||$_{0}$ denote a map from $H$ to $[0, \infty]$ such that if $\phi \in \mathscr{D},|\phi|_{0}$ $=|R \phi|$ and $|\langle\phi, K(\psi)\rangle| \leqq|\phi|_{0}|\psi|^{p-1}$.

THEOREM 6. If $U_{0}(t)$ satisfies condition (A7) below then $U_{0}(-t) U_{1}(t) \psi$ converges strongly to $\tilde{W}_{+} \psi$ for $\psi \in \mathscr{D}$.

(A7) For any $\phi \in H$, there is a $T(\phi)$ such that if $|t| \geqq T,\left|U_{0}(t) \phi\right|_{0}$ is bounded, i.e. there exists a constant $C(T, \phi)$ such that $\left|U_{0}(t) \phi\right|_{0} \leqq C$ for $|t| \geqq T$. 
Proof. Let $\psi_{t}=U_{0}(-t) U_{1}(t) \psi-\tilde{W}_{+} \psi$.

$$
\left\|\psi_{t}\right\| \leqq\left\|\tilde{W}_{+} \psi\right\|+\left(\|\psi\|^{2}+|\psi|^{p}\right)^{1 / 2}
$$

for all $t$. By Theorem 2, $\psi_{t}$ converges weakly to zero. Following the proof of Theorem 2, let $\phi \in H$, then

$$
\left|\left\langle\phi, \psi_{t}\right\rangle\right|<\int_{t}^{\infty}\left|\left\langle U_{0}(s) \phi, K\left(U_{1}(t) \psi\right)\right\rangle\right| d s .
$$

If $t \geqq T(\phi)$,

$$
\left|\left\langle\phi, \psi_{t}\right\rangle\right| \leqq C(T, \phi) \int_{t}^{\infty}\left|U_{1}(s) \psi\right|^{p-1} d s=O\left((1 / t)^{(p-2) / p}\right) .
$$

Thus $\psi_{t}$ tends strongly to zero by the previous lemma with $\varepsilon=(p-2) / p$.

REMARKS. (1) There is a similar result for $W_{-}$.

(2) It follows from the proof of Theorem 6 , that $\left|U_{0}(t) \phi\right|_{0}$ need not actually be bounded. What is needed is that $\left|U_{0}(t) \phi\right|_{0}$ be finite for all $t$ sufficiently large (depending on $\phi$ ) and not get large too rapidly with an estimate independent of $\phi$.

(3) Note that we have not used all the hypotheses on $K$. In particular, all that was used about $K$ was that it satisfy (A4), and that (1) has unique solutions for $\psi_{0} \in \mathbb{D}$ and $U_{1}(t)$ satisfies (a), (b), (c), (A2), (A3). It is not necessary that $K$ be everywhere defined for example.

2. The nonlinear wave equation. Let us consider the equation

$$
\square u=\Delta u-\partial^{2} u / \partial t^{2}=q u^{s}
$$

where $q=q\left(x_{1}, x_{2}, x_{3}\right)$ is positive, once differentiable and $s$ is an odd integer $\geqq 3$. We will assume also that $q$ is bounded and satisfies the differential inequality

$$
r q_{r} \leqq(s-3) q ; \quad\left(r=|x| \text { and } q_{r}=\partial q / \partial r\right) .
$$

Definition. $\mathscr{D}=C_{0}^{\infty}\left(R^{3}\right) \oplus C_{0}^{\infty}\left(R^{3}\right)$. I.e. $\psi=[f, g]$ is in $\mathscr{D}$ if $f$ and $g$ belong to $C_{0}^{\infty}\left(R^{3}\right)$.

Definition. If $\psi=[f, g]$ is in $\mathscr{D}$, let

$$
\|\psi\|^{2}=\int_{R^{3}}\left[|\nabla f|^{2}+g^{2}\right] d x .
$$

Let $H$ denote the completion of $\mathscr{D}$ with respect to \|\|$. H$ is a Hilbert space. We denote its inner product by $\langle$,$\rangle . It follows from the Sobolev inequality \|f\|_{6}$ $\leqq C\|\nabla f\|_{2}$ for $f \in C_{0}^{\infty}\left(R^{3}\right)$ that the components of an element $\psi \in H$ are functions. (\| $\|_{p}$ denotes the norm on $L_{p}\left(R^{3}\right)$.)

Let $U_{0}(t)$ be the strongly continuous one parameter group corresponding to the wave equation. For details see Thoe [4] or Lax and Phillips [1]. For $\psi=[f, g] \in H$ define $K(\psi)=\left[0,-q f^{s}\right]$. For $s=3, K$ is everywhere defined as a map from $H$ to $H$ and is locally Lipschitzian. For $s \geqq 3$ this is not so. We will assume $K$ satisfies the 
conditions of Remark 3, $\S 1$ however and also that $U_{1}(t) \psi \in \mathscr{D}$ for all $t$ if $\psi \in \mathscr{D}$ where $U_{1}(t)$ is defined by (1).

DEFINITION. If $\psi \in H$,

$$
|\psi|=\left(\int_{R^{3}} \frac{2}{s+1} q f^{s+1} d x\right)^{1 /(s+1)}
$$

where $\psi=[f, g]$. A standard energy equality states that if $\psi \in \mathscr{D}$,

$$
\left\|U_{1}(t) \psi\right\|^{2}+\left|U_{1}(t) \psi\right|^{s+1}=\|\psi\|^{2}+|\psi|^{s+1}
$$

for all $t$.

THEOREM 7. If $\psi \in \mathscr{D},\left|U_{1}(t) \psi\right|^{s+1}=O\left(t^{-2}\right)$ as $|t| \rightarrow \infty$.

Preof. See Strauss [2]. It is in this theorem that (5) is needed.

Definition. If $\psi=[f, g] \in H$,

$$
R \psi=((s+1) / 2)[g, 0]
$$

and

$$
|\psi|_{0}=\left(\int\left(\frac{s+1}{2}\right)^{s} q \dot{g}^{s+1} d x\right)^{1 /(s+1)}
$$

THEOREM 8. If $\phi, \psi \in H,|\langle\phi, K(\psi)\rangle| \leqq|\phi|_{0}|\psi|^{s+1}$.

Proof. When it makes sense,

$$
\langle\phi, K(\psi)\rangle=\int g q h^{s} d x,
$$

where $\phi=[f, g]$ and $\psi=[h, k]$. Thus

$$
\begin{aligned}
|\langle\phi, K(\psi)\rangle| & \leqq\left(\int q g^{s+1} d x\right)^{s /(s+1)}\left(\int q h^{s+1} d x\right) \\
& =|\phi|_{0}|\psi|^{s}=|R \phi||\psi|^{s},
\end{aligned}
$$

the last equality holding for $\phi \in \mathscr{D}$.

THEOREM 9. Any solution $\square u=0$ with data $\mathscr{D}$ remains there, i.e. $U_{0}(t) \mathscr{D} \subset \mathscr{D}$. Furthermore if $\psi \in \mathscr{D},\left|R U_{0}(t) \psi\right|^{s+1}=O\left(t^{-(s-1)}\right)$ as $|t| \rightarrow \infty$.

Proof. Let $U_{0}(t) \psi=\left[u(x, t), u_{t}(x, t)\right]$. Then

$$
\left|R U_{0}(t) \psi\right|^{s+1}=\int\left(\frac{s+1}{2}\right)^{s} q(x) u_{t}^{s+1}(x, t) d x .
$$

But $\int u_{t}^{2}(x, t) d x$ is bounded and $\left\|u_{t}(x, t)\right\|_{\infty}=O\left(t^{-1}\right)$. Thus

$$
\left|R U_{0}(t) \psi\right|^{s+1} \leqq\left(\int u_{t}^{2}(x, t) d x\right) \cdot\left\|u_{t}(x, t)\right\|_{\infty}^{s-1}\|q\|_{\infty}\left(\frac{s+1}{2}\right)^{s}=O\left(t^{-(s-1)}\right)
$$


The fact that $U_{0}(t) \mathscr{D} \subset \mathscr{D}$ is a standard regularity theorem for the wave equation. Letting $p=s+1$, we see that (A1), ., (A5) of $\$ 1$ are satisfied.

THEOREM 10. If $\phi, \psi$ are in $\mathscr{D},\left\langle\phi, U_{0}(t) \psi\right\rangle=0$ for all $t$ sufficiently large (and small).

Proof. $U_{0}(t) \psi$ is a detacined solution of the wave equation. Thus $u(x, t)=0$ in some forward cone and some backward cone. Let supp $f \cup$ supp $g \subset$ sphere about zero of radius $L$ where $\phi=[f, g]$. For $t$ sufficiently large or small this sphere is contained in the set where $u(x, t)$ vanishes.

THEOREM 11. If $\psi \in \mathscr{D}$ and $U_{1}(t) \psi=\left[u(x, t), u_{t}(x, t)\right]$, then for any $\varepsilon>0$,

$$
\int_{r<(1-\varepsilon)|t|}\left[|\nabla u|^{2}+u_{t}^{2}\right] d x=O\left(t^{-2}\right)
$$

as $|t| \rightarrow \infty$.

Proof. See Strauss [2]. (5) is also needed here.

THEOREM 12. If $\phi, \psi \in \mathscr{D},\left\langle\phi, U_{1}(t) \psi\right\rangle=O\left(t^{-1}\right)$ as $|t| \rightarrow \infty$.

Proof. Let $\phi=[f, g]$. Let $L$ be as in Theorem 10. Then

$$
\begin{aligned}
\left|\left\langle\phi, U_{1}(t) \psi\right\rangle\right| & =\left|\int\left[\nabla f \cdot \nabla u+g u_{\imath}\right] d x\right| \\
& =\left|\int_{|x| \leqq L}\left[\nabla f \cdot \nabla u+g u_{l}\right] d x\right| \\
& \leqq\|\phi\|\left(\int_{|x| \leqq L}\left[|\nabla u|^{2}+u_{t}^{2}\right] d x\right)^{1 / 2} .
\end{aligned}
$$

Thus for $|t|>2 L$,

$$
\left|\left\langle\phi, U_{1}(t) \psi\right\rangle\right| \leqq\|\phi\|\left(\int_{|x|<|t|}\left[|\nabla u|^{2}+u_{t}^{2}\right] d x\right)^{1 / 2}=O\left(t^{-1}\right)
$$

by Theorem 11 with $\varepsilon=\frac{1}{2}$.

Theorems 10 and 12 imply (A6).

The solutions of $\square u=0$ with data in $H$ are called finite energy solutions. The hypothesis of (A7) will be satisfied if for any $u(x, t)$ a finite energy solution

$$
\int q u_{t}^{s+1}(x, t) d x
$$

is eventually finite and bounded. In particular, for $q \equiv 1, u_{t}(x, t)$ must eventually be in $L_{s+1}\left(R^{3}\right)$. Since $L_{2} \nsubseteq L_{s+1}$ there are solutions for which this integral is infinite for $t$ near zero. There is no obvious reason why this should occur or for that matter any obvious counterexample. This result is of interest however since it replaces the problem of estimating solutions to the nonlinear equation with the apparently simpler problem of estimating solutions to the linear equation. 


\section{A. R. BRODSKY}

\section{BIBLIOGRAPHY}

1. P. Lax and R. Phillips, Scattering theory, Academic Press, New York, 1967.

2. W. Strauss, La décroisance asymptotique des solutions des équations d'onde nonlinéaires, C. R. Acad. Sci. Paris 256 (1963), 2749-2750.

3. - Les opérators d'onde pour des équations d'onde nonlinéaires, C. R. Acad. Sci. Paris 256 (1963), 5045-5046.

4. D. Thoe, Spectral theory for the wave equation with a potential term, Arch. Rational Mech. Anal. 22 (1966), 364-406.

\section{UNIVERSITY OF CALIFORNIA,}

los Angeles, California 